\title{
Femtosecond evolution of the pyrrole molecule excited in the near part of its UV spectrum
}

Cite as: J. Chem. Phys. 137, 064317 (2012); https://doi.org/10.1063/1.4742344

Submitted: 19 April 2012 . Accepted: 19 July 2012 . Published Online: 13 August 2012

Raúl Montero, Álvaro Peralta Conde, Virginia Ovejas, Marta Fernández-Fernández, Fernando Castaño, Javier R.

Vázquez de Aldana, and Asier Longarte

\section{ARTICLES YOU MAY BE INTERESTED IN}

Excited state non-adiabatic dynamics of pyrrole: A time-resolved photoelectron spectroscopy and quantum dynamics study

The Journal of Chemical Physics 142, 074302 (2015); https://doi.org/10.1063/1.4907529

Revisiting the relaxation dynamics of isolated pyrrole

The Journal of Chemical Physics 141, 014303 (2014); https://doi.org/10.1063/1.4885722

Ultrafast internal conversion dynamics of highly excited pyrrole studied with VUV/UV pump probe spectroscopy

The Journal of Chemical Physics 146, 064306 (2017); https://doi.org/10.1063/1.4975765

\section{Challenge us.}

What are your needs for periodic signal detection?

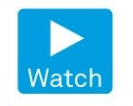

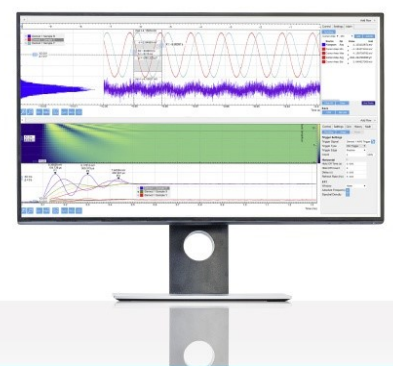

photoelectron spectroscopy 


\title{
Femtosecond evolution of the pyrrole molecule excited in the near part of its UV spectrum
}

\author{
Raúl Montero, ${ }^{1}$ Álvaro Peralta Conde,${ }^{1}$ Virginia Ovejas, ${ }^{1}$ Marta Fernández-Fernández, ${ }^{1}$ \\ Fernando Castaño, ${ }^{1}$ Javier R. Vázquez de Aldana, ${ }^{2}$ and Asier Longarte ${ }^{1, a)}$ \\ ${ }^{1}$ Departamento de Química Física, Universidad del País Vasco (UPV/EHU), Apart. 644, \\ 48080 Bilbao, Spain \\ ${ }^{2}$ Departamento de Física Aplicada, Facultad de Ciencias, Universidad de Salamanca, \\ Salamanca 37008, Spain
}

(Received 19 April 2012; accepted 19 July 2012; published online 13 August 2012)

\begin{abstract}
The evolution of the isolated pyrrole molecule has been followed after excitation in the 265-217 $\mathrm{nm}$ range by using femtosecond time delayed ionization. The transients collected in the whole excitation range show the vanishing of the ionization signal in the femtosecond time scale, caused by the relaxation along a $\pi \sigma^{*}$ type state $\left(3 \mathrm{~s} \mathrm{a}_{1} \leftarrow \pi 1 \mathrm{a}_{2}\right)$, which is the lowest excited electronic state of the molecule. This surface is dissociative along the NH bond, yielding a $15 \pm 3$ fs lifetime that reflects the loss of the ionization cross-section induced by the ultrafast wavepacket motion. Although a weak $\pi \sigma^{*}$ absorption is detected, the state is mainly reached through internal conversion of the higher bright $\pi \pi^{*}$ transitions, which occurs with a $19 \pm 3 \mathrm{fs}$ lifetime. In addition to its resonant excitation, the intense $\pi \pi^{*}$ absorption extending in the 220-190 nm interval is also out-of-resonance populated at energies far to the red from its absorption onset. This coherent adiabatic excitation of the $\pi \pi^{*}$ transition should follow the excitation pulse (coherent population return effect), but instead the system relaxes toward the lower $\pi \sigma^{*}$ surface through a conical intersection during the interaction time, leading to the population of $\pi \sigma^{*}$ state at wavelengths as long as $265 \mathrm{~nm}$. According to the observed behavior, the time evolution of the system in the full excitation range studied is modeled by a coherent treatment that provides key insights on the photophysical properties of the molecule. (C) 2012 American Institute of Physics. [http://dx.doi.org/10.1063/1.4742344]
\end{abstract}

\section{INTRODUCTION}

Pyrrole, a heteroatomic aromatic molecule with a five member ring containing a nitrogen atom, is a quite common compound with important chromophoric properties. ${ }^{1}$ In biology, substituted pyrroles are the constituent units of porphyrins based pigments and other relevant compounds, being also closely related to the pyrimidinic DNA bases. Although its apparent simplicity, the photoexcitation of pyrrole in the near UV range gives rise to a variety of photophysical processes that, at least when solvated by water, include electron and proton transfer phenomena. ${ }^{2}$

In the last years, a very successful effort has been devoted to determine the electronic states and energy pathways involved in the relaxation of simple aromatic chromophores, opening the door to the understanding of more complicated systems, and even the contribution of the surrounding environment. A decisive aspect in unraveling these mechanisms has been the recognition of the role of the $\pi \sigma^{*}$ type transitions. ${ }^{3,4}$ These excitations, usually of dark nature, give rise to repulsive potentials along the $\mathrm{X}-\mathrm{H}(\mathrm{X}$ : $\mathrm{N}$ or $\mathrm{O})$ stretching coordinates. The evolution along these surfaces, which can be reached indirectly by coupling to other bright states, induces different photophysical processes including, fragmentation and internal conversion (IC) to the ground state through

a) Author to whom correspondence should be addressed: Electronic mail: asier.longarte@ehu.es. Telephone: +34 946018086. Fax: +34 946013500. non-adiabatic crossings. ${ }^{3,5,6}$ An important number of time and frequency resolved experimental works, together with detailed theoretical descriptions, have permitted to identify and characterize $\pi \sigma^{*}$ states in some simple but fundamental aromatic chromophores as indole, ${ }^{3,7-13}$ phenol, ${ }^{6,14,15}$ aniline ${ }^{16-19}$ or imidazole, ${ }^{20-24}$ among others. From these studies, a general picture where the photopysical properties are the result of the strong interaction between the $\pi \pi^{*}$ and $\pi \sigma^{*}$ states seems to emerge.

Pyrrole is an excellent model to gain a further understanding on the dynamical processes induced by the presence of $\pi \sigma^{*}$ states, since the lowest singlet excited state belongs to this category of transitions. It is a Rydberg ${ }^{1} \mathrm{~A}_{2}$ state (according to the $\mathrm{C}_{2 \mathrm{~V}}$ planar symmetry of the molecule) that results of the promotion of an electron from the $1 \mathrm{a}_{2}$ aromatic orbital to the $3 \mathrm{sa}_{1}$ orbital of Nitrogen, which acquires $\sigma^{*}$ character along the $\mathrm{N}-\mathrm{H}$ coordinate. ${ }^{3,25}$ Although the transition is dipole moment forbidden, ${ }^{26-28}$ it is thought to be responsible of the weak longest wavelength portion of the UV absorption spectrum of pyrrole, starting around $250 \mathrm{~nm}(4.96 \mathrm{eV}),{ }^{29,30}$ by gaining some intensity through vibronic coupling. ${ }^{28,31}$ Immediately above this band, and centered around $210 \mathrm{~nm}(5.90 \mathrm{eV})$, the spectrum shows an intense unresolved feature that is attributed to several overlapping excitations. $^{28,30}$ According to experimental observations, ${ }^{30}$ and further confirmed by theoretical predictions, ${ }^{28}$ two $\pi \pi^{*}$ transitions, $2^{1} \mathrm{~A}_{1} \leftarrow \mathrm{X}^{1} \mathrm{~A}_{1}$ and $1^{1} \mathrm{~B}_{2} \leftarrow \mathrm{X}^{1} \mathrm{~A}_{1}$ are mainly 
responsible for this absorption, being the later the one that carries the most oscillator strength. Additionally, three Rydberg transitions $2^{1} \mathrm{~A}_{2}\left(3 \mathrm{p}_{\mathrm{z}}\right) \leftarrow \mathrm{X}^{1} \mathrm{~A}_{1}, 1^{1} \mathrm{~B}_{1}\left(3 \mathrm{p}_{\mathrm{y}}\right) \leftarrow \mathrm{X}^{1} \mathrm{~A}_{1}$, and $2^{1} \mathrm{~B}_{2} \leftarrow \mathrm{X}^{1} \mathrm{~A}_{1}$ are also predicted to contribute to a minor extent. $^{28}$

The spectroscopic and dynamical behavior that emerges from this electronic structure has been probed in different experimental studies. The attempts made to record REMPI $(1+1)$ spectrum across the absorption region described above did not show any feature, pointing to an ultrashort lifetime of the molecule when excited at those energies. ${ }^{32}$ Experiments based in kinetic energy resolved detection of $\mathrm{H}$ fragments have provided key information regarding the photochemical processes the molecule undergoes after excitation in the 250-200 nm range. $31,33-35$ The formation of $\mathrm{H}$ atoms is observed in the whole range of studied wavelengths, presenting a kinetic energy distribution compatible with at least two elimination pathways: a fast $\mathrm{N}-\mathrm{H}$ bond fission along the repulsive $\pi \sigma^{*}$ potential and a decomposition mediated by the IC to the ground state. ${ }^{31,33}$ A femtosecond time resolved study has supported these data by finding for the $\mathrm{H}$ atom formation two lifetimes of $\sim 100$ fs and $\sim 1$ ps after exciting pyrrole at $250 \mathrm{~nm}^{36}$ The calculated potential energy surfaces provide an explanation for these observations by characterizing the non-adiabatic couplings between the involved states. The dissociative $\pi \sigma^{*}$ state is predicted to cross the ground state potential curve at $\mathrm{N}-\mathrm{H}$ distances about $1.8 \AA$, forming a conical intersection (CI) promoted by the out of plane bending of the $\mathrm{N}-\mathrm{H}$ bond that triggers the fast IC to the ground state. In this picture, the $\pi \sigma^{*}$ state may be also reached by ultrafast IC from the above optically active states, since the dynamics along this surface fully determines the photophysical behavior of the molecules. . $^{3,37,38}$

Herein, we present a time resolved study with ultrafast resolution on the pyrrole relaxation dynamics in the 265-217 nm excitation range. Previous attempts to record the dynamics of the system directly on the parent ion did not yield conclusive results, probably due to the lack of the required time resolution. ${ }^{36}$ Our work provides a clear view of the ultrafast relaxation induced by the $\pi \sigma^{*}$, measuring two lifetimes of $19 \pm 3$ and $15 \pm 3 \mathrm{fs}$, which are associated with the IC from the bright dominant ${ }^{1} \mathrm{~B}_{2} \pi \pi^{*}$ state and the propagation of the wavepacket on the $\pi \sigma^{*}$ state, respectively. The study also explores the consequences of the adiabatic excitation of the system when using femtosecond pulses for the preparation of the molecules in the targeted electronic states, revealing the key implication of this type of coherent phenomena. The collected data reveal that the bright ${ }^{1} \mathrm{~B}_{2} \pi \pi^{*}$ state is transiently populated at excitation wavelengths far away from resonance, by an adiabatic coherent excitation process that follows the excitation pulse. The ultrafast relaxation during the interaction time, mediated by a CI, provides an efficient way to reach the $\pi \sigma^{*}$ state. The recorded transients are fit employing a coherent model that provides a comprehensive view of the dynamical processes pyrrole undergoes after excitation by ultrashort light pulses.

\section{METHODS}

\section{A. Experimental procedures}

The evolution of the electronic excitation in isolated pyrrole molecules was tracked with femtosecond resolution by time delayed ionization. The measurements were carried out in linear time of flight mass-spectrometer. The vapor pressure of pyrrole (Aldrich, 98\%) was mixed with 1-2 atm of Ar and expanded through an electromagnetically controlled valve (Parker, Series 9), to form a supersonic expansion that was collimated by a skimmer at the entrance of the ionization region. The ions formed in the interaction with the pump and probe beams were accelerated toward a dual multichannel plate $(18 \mathrm{~mm})$ detector located at the end of the $80 \mathrm{~cm}$ flight tube. The mass spectra were monitored by a digital scope, while the signals of the ions of interest were integrated by up to three boxcars (Stanford SR250), and stored in a personal computer after digitalization by an A/D converter.

Femtosecond laser pulses were generated by a commercial Ti:Saphire oscillator-regenerative amplifier (Coherent Legend) system $(1 \mathrm{kHz}, 4.0 \mathrm{~mJ}, 35 \mathrm{fs}$ pulses @ $800 \mathrm{~nm})$. A portion of the amplifier output at $800 \mathrm{~nm}$ was used as a probe beam. The generation of the pump wavelengths in the studied excitation range was achieved through two different interactions. In the $265-235 \mathrm{~nm}$ interval, pump pulses were produced by the second harmonic of the sum frequency generation of the signal of an optical parametric amplifier (OPA), and the $800 \mathrm{~nm}$ fundamental beam. To generate light below $235 \mathrm{~nm}$ the sum of the OPA signal and the $800 \mathrm{~nm}$ fundamental is mixed with $400 \mathrm{~nm}$ radiation in a $100 \mu \mathrm{m}$ thick Beta Barium Borate (BBO) crystal cut at the $\theta=63.7^{\circ}$ angle. This process provides a beam that can be tuned in the 235-217 nm interval. Detailed information on the method is provided in the supplementary material. ${ }^{39}$ The pump pulses travelled through a prism compressor before going into the spectrometer, achieving durations at the interaction region of 60 and 80 fs in the 265-235 and 235-217 nm intervals, respectively. The non-resonant $1+4^{\prime}$ ionization signal of ethylene was collected simultaneously to the pyrrole ion in order to establish the zero delay time $\Delta \mathrm{t}=0$ and the cross-correlation function. The latter yielded values of 60 and $80 \mathrm{fs}$ for the low and high energy portion of the excitation spectrum, respectively. Regarding the intensities used in the experiments, the pump was adjusted to avoid saturation effects and multiphoton processes (no measurable ion signal is observed in the absence of the probe beam), while the probe was kept around $10^{12} \mathrm{~W} / \mathrm{cm}^{2}$. The relative polarization of pump and probe beams was set at magic angle configuration.

\section{B. Calculations}

In order to estimate the pyrrole dissociation rates after direct excitation to the ${ }^{1} \mathrm{~B}_{2} \pi \sigma^{*}$ state, the potential energy surface (PES) of this state was mapped by a relaxed scan along the $\mathrm{NH}$ stretching coordinate, carried out with the TD/B3LYP/6-311++G(d,p) method implemented in the GAUSSIAN 09 package. ${ }^{40}$ The ground neutral and ionic PES 


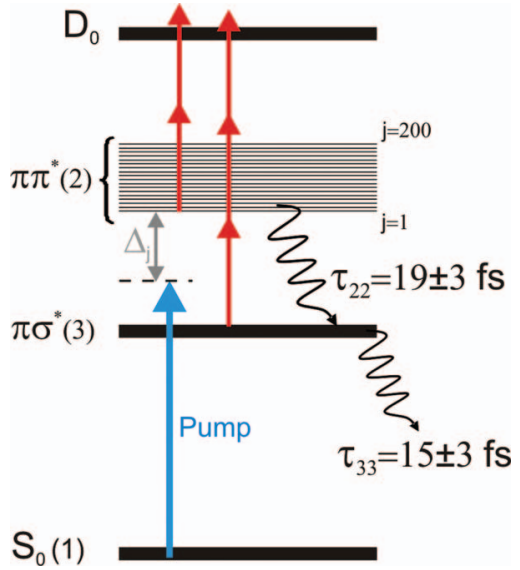

FIG. 1. Schematics of the involved states and processes included to model the pyrrole dynamical behavior. The $\pi \pi^{*}$ absorption is represented by a set of $\mathrm{N}=200$ equally spaced $(\delta \mathrm{E})$ virtual states. The detuning of the excitation laser relative to the individual $\mathrm{j}$ states is given by $\Delta_{\mathrm{j}} . \tau_{22}$ is the internal conversion of the $\pi \pi^{*}$ state and $\tau_{33}$ the ultrafast dynamics along the $\pi \sigma^{*}$ state.

were also scanned along the $\mathrm{NH}$ stretching coordinate at B3LYP/6-311++G(d,p) level.

A coherent model based on the solution of the optical Bloch equations was employed to fit the experimental transients. ${ }^{41}$ The model, sketched in Fig. 1, embraces the three relevant electronic states in the explored excitation energy: ground (1), $\pi \sigma^{*}$ (3), and the $\pi \pi^{*}$ states (2). In order to account for its broad absorption spectrum, the $\pi \pi^{*}$ state is represented by a quasi continuum of $\mathrm{j}=200$ identical (same oscillator strength and ionization cross-section is assumed), and equally spaced $\left(\delta \mathrm{E}=16.7 \mathrm{~cm}^{-1}\right)$ discrete states. The detuning of the laser central wavelength with respect to each state of the continuum is expressed as $\Delta_{\mathrm{j}}$. Considering that the ground state is barely perturbed, which is a good approximation in the case of excitation with broadband laser pulses, ${ }^{42}$ the population of the $\pi \pi^{*}$ state is given by the sum of the populations of the individual states.

Due to its very low oscillator strength, the $\pi \sigma^{*}$ state is not considered to be populated by direct excitation, it only receives population by conversion $\left(\Gamma_{22}\right)$ of the upper $\pi \pi^{*}$ state. The dynamics along the $\pi \sigma^{*}$ surface is given by the $\Gamma_{33}$ constant. The resulting temporal evolution of the populations and coherences can be written as

$$
\begin{gathered}
\dot{\rho}_{11}=\frac{i}{2} \Omega_{0} g(t)\left(\rho_{12 j}-\rho_{21 j}\right), \\
\dot{\rho}_{22 j}=-\frac{i}{2} \Omega_{0} g(t)\left(\rho_{12 j}-\rho_{21 j}\right)-\Gamma_{22} \rho_{22 j}, \\
\dot{\rho}_{33 j}=\Gamma_{22} \rho_{22 j}-\Gamma_{33} \rho_{33 j}, \\
\dot{\rho}_{12 j}=\frac{i}{2} \Omega_{0} g(t)\left(\rho_{22 j}-\rho_{11 j}\right)-\left(\frac{\Gamma_{22}}{2}-i 2 \Delta_{j}\right) \rho_{21 j}, \\
\rho_{22}=\sum_{j=0}^{N} \rho_{22 j} ; \rho_{33}=\sum_{j=0}^{N} \rho_{33 j},
\end{gathered}
$$

where $g(t)$ is the is the Gaussian time profile of the of the pump laser electric field and $\Omega_{0}$ the Raby frequency.
The molecular response function employed to fit the transients is obtained after multiplying the time dependent populations, by the ionization cross sections $\left(\gamma_{22}\right.$ and $\left.\gamma_{33}\right)$. The probe pulse can be considered instantaneous since it is considerably shorter than the pump.

From the fitting of all the excitation wavelengths, a single set of $\Gamma_{22}$ and $\Gamma_{33}$ constants, and $\gamma_{22}$ and $\gamma_{33}$ ionization cross sections, is extracted. Only in the case of the $252 \mathrm{~nm}$ excitation, the derived $\rho_{33}$ population is artificially increased to account for the possibility, not included in the model, of reaching the $\pi \sigma^{*}$ by direct excitation (see below).

\section{RESULTS}

The time evolution of the pyrrole ion signal was recorded after excitation in the 265-217 $\mathrm{nm}$ range, while probing in all cases with $800 \mathrm{~nm}$ light. At the pump-probe conditions employed, the mass spectrum did not show appreciable ionic fragments. In the spectral region covered by the excitation, pyrrole exhibits, as stated above, two main absorptions: a very weak band around $240 \mathrm{~nm}$ and the strong unresolved feature starting at $220 \mathrm{~nm}$ and extending beyond $190 \mathrm{~nm}$. The former is attributed to the ${ }^{1} \mathrm{~A}_{2} \pi \sigma^{*}$ state, while the latter comprises a number of excitations, being the main one the $1 \mathrm{~B}_{2} \pi \pi^{*}$ state. From now on we will refer to this band simply as the $\pi \pi^{*}$. Figure 2 summarizes the transients collected along this whole range. The four transients, plotted together with the ethylene non-resonant signal simultaneously recorded, present a similar appearance. The signal vanishes completely after less than $200 \mathrm{fs}$, but a detailed analysis, carried out in all cases with the same theoretical model described above, reveals the occurrence of different processes in the femtosecond time scale.
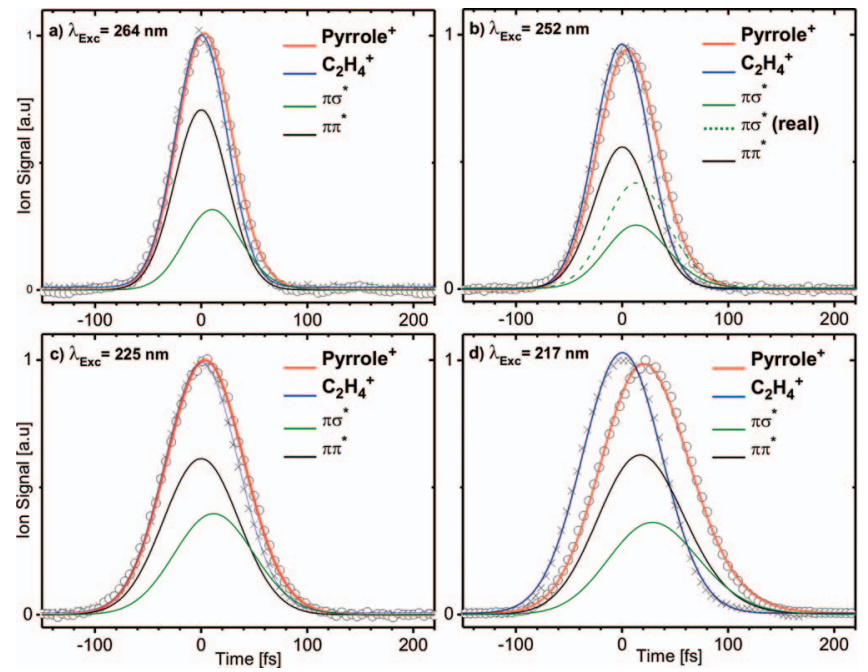

FIG. 2. Transients collected at the pyrrole ${ }^{+}$and the ethylene ${ }^{+}\left(1+4^{\prime}\right.$ nonresonant ionization signal used as reference) mass channels by exciting at the indicated wavelength and probing with $800 \mathrm{~nm}$ laser pulses. The circles (pyrrole) and crosses (ethylene) correspond to the experimental data, while the red and blue lines are their best obtained fittings. The black and green solid lines are the individual contributions of the $\pi \pi^{*}$ and $\pi \sigma^{*}$ states, respectively, predicted by the model. In (b), the dashed green line represents the contribution of the $\pi \sigma^{*}$ state employed to fit the transient, which was increased relative to the model prediction (solid line) to account for the resonant excitation not included in the model. 
This ultrafast dynamics is reflected in the collected transients as the position shift and width increment that the pyrrole ion signal experiences, relative to the ethene reference signal, at all the excitation wavelengths. Consequently, in order to derive any meaningful information from the collected data, a precise determination of these parameters is mandatory. To support the accuracy of the experiments carried out, a table containing detailed information on the shifts and width increments measured, together with their associated errors, is included in the supplementary material (Table 1s). In the same sense, we have added Figure 5s showing five decays of the pyrrole ion recorded with slightly different experimental conditions over a period of several days, which can provide an impression of the data quality. The interpretation of the observed dynamical behavior and its dependence on the excitation wavelength relies on the model sketched in Fig. 1. The need of using a coherent model instead the more usual rate equation approach is triggered by the observation of a $15 \pm 3$ fs lifetime at excitation energies below the first electronic absorption of the molecule. This finding, which will be explained in terms of the off-resonance coherent excitation of the $\pi \pi^{*}$ state of the molecule, cannot be reproduced without a quantum interpretation. The application of the coherent treatment to the transients shown in Figure 2 yields two lifetimes common to all the excitation wavelengths: $\tau_{22}=19 \pm 3 \mathrm{fs}$ and $\tau_{33}=15 \pm 3$ fs.

Trace 2a, recorded at the $264 \mathrm{~nm}$ pump wavelength, corresponds to the excitation of the molecule at energies below the first singlet excitation, the ${ }^{1} \mathrm{~A}_{2} \pi \sigma^{*}$ state. The individual contributions to the ionization signal of the $\pi \sigma^{*}$ and the $\pi \pi^{*}$ states are shown in the graph, together with the experimental data and the total ion signal (sum of ions formed at the $\pi \sigma^{*}$ and the $\pi \pi^{*}$ states) derived from the model. While the $\pi \sigma^{*}$ state decays with the $\tau_{33}=15 \pm 3$ fs lifetime, the $\pi \pi^{*}$ signal appears as Gaussian like component that matches the ethylene cross-correlation signal. As explained in detail below, this behavior is a consequence of being coherently out of resonance excited, which leads to an adiabatic evolution that follows the excitation pulse. However, it is important to remark that depopulation rate of this state derived from the fitting is given by the $\tau_{22}=19 \pm 3$ lifetime, which modulates the relative amplitudes of the $\pi \pi^{*}$ and $\pi \sigma^{*}$ transient signals.

The transient recorded at $252 \mathrm{~nm}$, on resonance with the weak absorption of the spectrum attributed to the ${ }^{1} \mathrm{~A}_{2} \pi \sigma^{*}$, is shown in trace $2 \mathrm{~b}$. The same evolution of the $\pi \sigma^{*}$ and $\pi \pi^{*}$ states is observed, although the fitting requires an increment of the $\tau_{33}$ component weight with respect to that used for the $264 \mathrm{~nm}$ excitation (see below).

Trace 2c depicts the transient collected at $225 \mathrm{~nm}$, near the onset of the strong absorption of the $\pi \pi^{*}$ band. The system mimics the behavior observed at the $264 \mathrm{~nm}$ excitation, while showing compared to the $252 \mathrm{~nm}$ signal, a considerable reduction of the $\tau_{33}$ lifetime weight.

Finally, trace $2 \mathrm{~d}$ corresponds to the signal registered at the highest excitation energy, on resonance with the strong $\pi \pi^{*}$ absorption. In this case, the transient reflects again the time dependent population of both $\pi \pi^{*}$ and $\pi \sigma^{*}$ states. However, while the evolution of the $\pi \sigma^{*}$ state is again described by $\tau_{33}=15 \pm 3 \mathrm{fs}$, the population of the $\pi \pi^{*}$ state decays at this wavelength with the $\tau_{22}=19 \pm 3$ fs lifetime. The explicit observation of $\tau_{22}$ is, as discussed below, a consequence of the resonant excitation of the $\pi \pi^{*}$ state.

A detailed explanation of the observed temporal evolution follows, which for the sake of clarity, is separated for the different excitation wavelength.

\section{A. $\lambda_{\mathrm{Exc}}=264 \mathrm{~nm}$}

The model used to fit the transients collected at all the excitation wavelengths grounds on the coherent excitation of the $\pi \pi^{*}$ state, while due to the low oscillator strength, the coupling of the ground and the $\pi \sigma^{*}$ state through the laser interaction is neglected. In this coherent view, when excited out of resonance, the $\pi \pi^{*}$ evolves adiabatically following the excitation pulse. Thus, the population reaches the $\pi \pi^{*}$ state transitorily, only during the interaction with the laser, returning back to the ground state after it, unless the coherence is lost. ${ }^{43}$ In these conditions, the contribution of the $\pi \pi^{*}$ population to the ionization signal appears as a 0 fs component that mimics the cross-correlation function. The occurrence of this effect, named coherent population return (CPR) (Refs. 44 and 45) has been reported previously when broadband femtosecond laser pulses are employed to excite molecules in the vicinity of strongly allowed transitions. ${ }^{42,46}$ In order to further illustrate this behavior, a simulation of the excited state evolution in a two level system excited on and off resonance, has been included in the supplementary material (Figures $6 \mathrm{~s}$ and $7 \mathrm{~s}){ }^{39}$

A careful analysis of the dependence of the signal intensity at $\Delta \mathrm{t}=0$ on the detuning with respect to the $\pi \pi^{*}$ band was carried out, to test the validity of the CPR interpretation. For the case of a barely perturbed two-level system evolving adiabatically, the excited state population can be approximated by the expression: ${ }^{47}$

$$
P_{2}=\frac{\Omega^{2}(t)}{4 \Delta^{2}},
$$

being $\Omega(t)$ the Raby frequency and $\Delta$ the detuning of the excitation from resonant condition. Figure 3 shows the fit of the pump only $(1+1)$ signal (weighted by the laser intensity) to

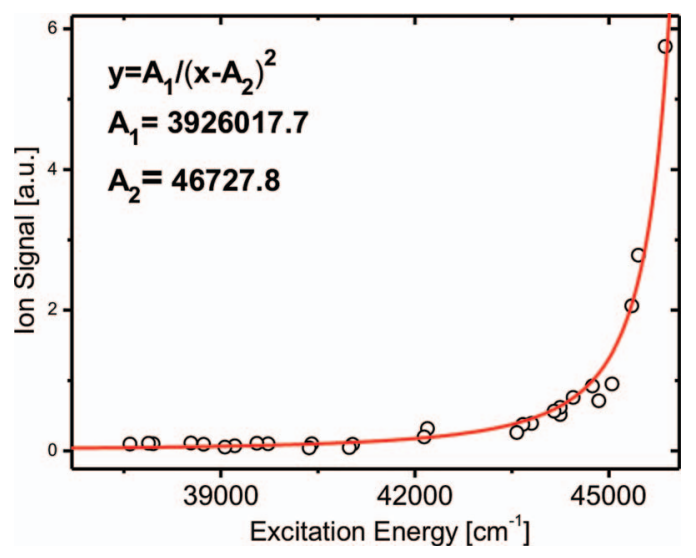

FIG. 3. Dependence of the pyrrole ${ }^{+}$pump only $(1+1)$ signal on the excitation wavelength. The circles are the experimental points while the solid line is the fit to the indicated function. 
a function of the form:

$$
y=\frac{A_{1}}{\left(x-A_{2}\right)^{2}} .
$$

Assuming a constant ionization cross-section at all the excitation wavelengths, the $\mathrm{A}_{2}$ parameter should yield the position of the band responsible of the CPR signal. The value obtained, $46728 \mathrm{~cm}^{-1}(214 \mathrm{~nm})$, agrees reasonably with the maximum of the $\pi \pi^{*}$ absorption, ${ }^{28,30}$ proving the nature of the observed ionization signal at $\Delta \mathrm{t}=0$.

The above interpretation, based in the out of resonance coherent excitation of the $\pi \pi^{*}$ state, also explains the origin of the $\tau_{33}=15 \pm 3$ fs component observed at all the studied excitation wavelengths, even below the first singlet electronic state. We attribute $\tau_{33}$ to ultrafast dynamics along the $\pi \sigma^{*}$ surface. Although we prefer to postpone the discussion on the lifetime for Sec. III B, an explanation on how the $\pi \sigma^{*}$ is reached seems mandatory. The population of this surface takes place by IC of the higher $\pi \pi^{*}$ state, which, as stated above, is coherently excited during the pump pulse. The $\pi \pi^{*}$ transitory population follows the pump pulse temporal evolution and the IC acts as dephasing process that does not alter the Gaussian profile of this signal, only the intensity is affected (see supplementary material Figure $6 \mathrm{~s}$ ) ${ }^{39}$ The decay of the $\pi \pi^{*}$ state will show directly the rate of the IC, $\tau_{22}$ $=19 \pm 3 \mathrm{fs}$, when it is resonantly excited at $217 \mathrm{~nm}$ (see below).

\section{B. $\lambda_{\mathrm{Exc}}=252 \mathrm{~nm}$}

The transient recorded at $252 \mathrm{~nm}$ (Fig. 2(b)) exhibits the same dynamics already observed at the lower excitation wavelength, holding for it the argumentation offered above. However, there are some subtle singularities that need a deeper analysis. Figure 4 shows a comparison between the experimental decays recorded at 264 and $252 \mathrm{~nm}$, together with the ethylene non-resonant signal. The $252 \mathrm{~nm}$ transient appears to be shifted to longer delays, which requires an increment of the relative weight of the $\tau_{33}=15 \pm 3$ fs component for a correct fitting. This effect has been only observed at this excitation wavelength, and we believe that is caused by the

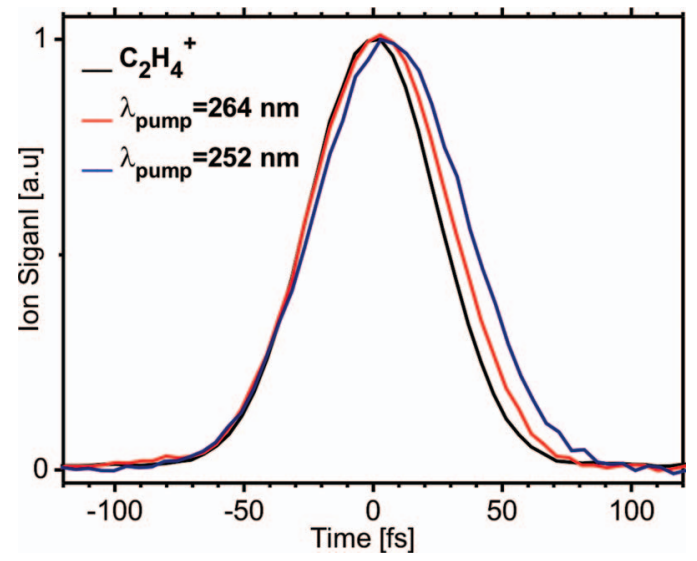

FIG. 4. Comparison between the transients collected at pyrrole ${ }^{+}$mass channel exciting at 264 (red) and 251 (blue) nm. The non-resonant signal of ethylene (black) is included to determine the cross-correlation function and the zero delay time. fraction of molecules reaching the $\pi \sigma^{*}$ by resonant excitation. The possibility of preparing this state by single photon absorption from the ground state has been considered in previous studies. ${ }^{31,33}$ Although the work by Beames et al. ${ }^{32}$ reports no sign of absorption (other than some lines attributed to multiphoton excitation of Rydberg states) in the REMPI spectrum across this region, theoretical studies predict a weak absorption of the $\pi \sigma^{*}$ state around $250 \mathrm{~nm}$. Additional evidences of this transition are provided by the work by Wei et al., ${ }^{31}$ which detects the formation of fast hydrogen atoms, attributed to direct dissociation along the $\pi \sigma^{*}$ after excitation at $243 \mathrm{~nm}$.

In order to evaluate the $\pi \sigma^{*}$ state absorption, a simulation keeping all the fitting parameters fixed while providing some oscillator strength to the $\pi \sigma^{*}$ state was carried out. From the calculation, a rough value of 0.0027 was derived for the $\pi \sigma^{*} / \pi \pi^{*}$ oscillator strength ratio, which seems a reasonable value according to the experimental observations.

Consequently, the $15 \pm 3$ fs component measured at the $252 \mathrm{~nm}$ excitation corresponds to the dynamics on the $\pi \sigma^{*}$ state simultaneously prepared via direct excitation and internal conversion from the higher $\pi \pi^{*}$ state adiabatically excited, showing both pathways the same or a very close (in the accuracy of the experiment) lifetime.

It is worth noting that in Figure 2(b) the two green curves corresponding to direct excitation and indirect population via IC of the $\pi \sigma^{*}$ state, show exactly the same time profile: a Gaussian curve centered at $\Delta \mathrm{t}=0$. Although the $\pi \sigma^{*}$ state is in one of the cases populated indirectly via $\pi \pi^{*}$ state, the later is out-of-resonance coherently prepared following the excitation pump pulse. Consequently, the $\pi \sigma^{*}$ state population grows as the excitation proceeds, without measurable delay time with respect to it. To illustrate this result, we have included in the supplementary material (Figure $6 \mathrm{~s}$ and $7 \mathrm{~s}$ ) a simulation for the on and off-resonance excitation of a twolevel system coupled to a third state. Figure 6s (upper) shows how when the state 2 is out-of-resonance prepared, the population of state 3 raises following the excitation pulse. However, for the on-resonance case, the state 3 population grows with the expected time constant that reflects the internal conversion from the upper state 2 .

In order to rationalize the ultrafast rate observed for the dynamics along the $\pi \sigma^{*}$ state, its PES was mapped along the $\mathrm{NH}$ stretching coordinate, and compared with those calculated for the ground neutral ${ }^{1} \mathrm{~A}_{1}$ and ${ }^{2} \mathrm{~A}_{2}$ ion states (Fig. 5). The reduction of the system to a single coordinate seems in this case a reasonable assumption, in particular, if we consider that the NH stretch is the fastest vibration. The calculation shows the repulsive character of the $\pi \sigma^{*}$ state and permits to make an estimation of the time required to leave the window for ionization, taken as the energy region where three $800 \mathrm{~nm}$ photons are enough to ionize the molecule (see Fig. 5). The simple application of the energy conservation, assuming a direct excitation from the ground zero point energy at the equilibrium geometry, to the $\pi \sigma^{*}$ calculated potential, yields around $10 \mathrm{fs}$ to leave the ionization region. This number can be directly compared with the $15 \pm 3$ fs extracted from the transients. 


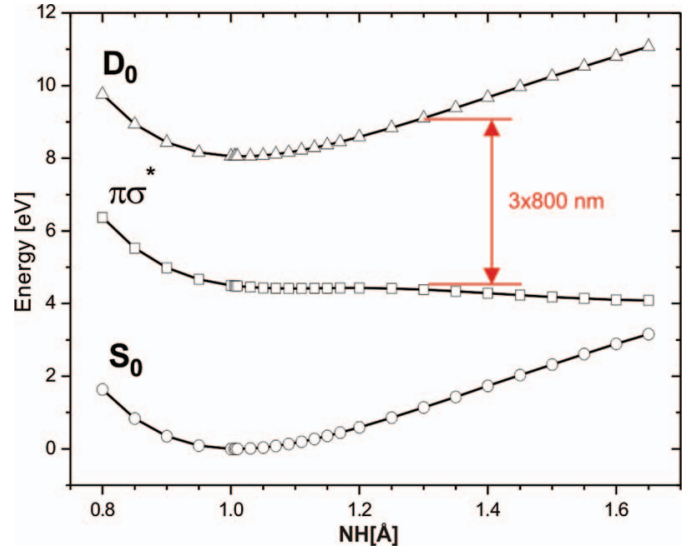

FIG. 5. Potential energy profiles of the ground neutral (circles), $\pi \sigma^{*}$ (squares), and ground ionic (triangles) states of pyrrole, as a function of the $\mathrm{N}-\mathrm{H}$ bond distance. The $\pi \sigma^{*}$ geometries and energies where optimized at TD/B3LYP/6-311++G(d,p) level, while the ground states at B3LYP/6$311++\mathrm{G}(\mathrm{d}, \mathrm{p})$. The energy corresponding to three $800 \mathrm{~nm}$ photons is indicated by the red arrow.

\section{C. $\lambda_{\mathrm{Exc}}=225 \mathrm{~nm}$}

The behavior of the system at this wavelength mimics that already observed at $264 \mathrm{~nm}$. The excitation is shifted out of the weak resonance of the $\pi \sigma^{*}$ state and cannot reach the higher $\pi \pi^{*}$ absorption. Accordingly, as discussed in Sec. III A, the observed dynamics correspond to the $\pi \sigma^{*}$ state reached via IC of the adiabatically prepared $\pi \pi^{*}$ state. The smaller detuning from the resonance leads to a larger amount of population being out-of-resonance excited through the $\pi \pi^{*}$ state absorption. However, the dynamics of the system is the same at all the off-resonance wavelengths, except at $252 \mathrm{~nm}$, where direct excitation of the $\pi \sigma^{*}$ state occurs simultaneously.

The transients collected at 264 and $225 \mathrm{~nm}$ only differ on the cross-correlation function. The higher dispersion the $225 \mathrm{~nm}$ pulses undergo broadens their temporal profile, resulting in a poorer time resolution. The longer pump pulses extend the interaction with the $\pi \pi^{*}$ state, increasing the time for the $\pi \sigma^{*} \leftarrow \pi \pi^{*}$ internal conversion. This fact is reflected by the larger $\pi \sigma^{*} / \pi \pi^{*}$ contribution ratio observable at $225 \mathrm{~nm}$ with respect to that at $264 \mathrm{~nm}$.

\section{D. $\lambda_{\mathrm{Exc}}=217 \mathrm{~nm}$}

The excitation of ${ }^{1} \mathrm{~B}_{2} \pi \pi^{*}$ state yields transients notably different to those collected at lower energies. The evolution of the $\pi \pi^{*}$ state is described now by the $\tau_{22}=19 \pm 3$ fs lifetime, which is attributed to the ultrafast IC of the resonantly prepared $\pi \pi^{*}$ toward the lower $\pi \sigma^{*}$ state. The conversion process had already been derived from the out of resonance measurements, but the corresponding lifetime had not been explicitly observed in the time profile of the $\pi \pi^{*}$ state population. After conversion, the system evolves along the dissociative $\pi \sigma^{*}$ giving rise to the same $\tau_{33}$ lifetime.

\section{DISCUSSION}

The literature accumulates a great number of experimental and theoretical works on the photochemistry of pyrrole, which can be compared with the present results to clarify some important aspects of the problem. Our data yield a $\tau_{33}=15 \pm 3$ fs lifetime for the dynamics along the $\pi \sigma^{*}$ surface when prepared by direct excitation or by conversion from the upper $\pi \pi^{*}$ state. The work by Lippert et al. ${ }^{36}$ has studied the $\pi \sigma^{*}$ state mediated formation of $\mathrm{H}$ fragments with time resolution at the femtosecond scale, reporting two lifetimes of $100 \mathrm{fs}$ and $1.1 \mathrm{ps}$ at $250 \mathrm{~nm}$ excitation. These components have been assigned, respectively, to the direct and after IC to the ground state dissociation pathways, being the later mediated by the $\pi \sigma^{*} / \mathrm{S}_{0} \mathrm{CI}$. Although the lifetime extracted from our experiment seems in principle considerably shorter, two aspects need to be considered. While the $\mathrm{H}$ atom formation lifetime corresponds essentially to the time the system spends in reaching the $\pi \sigma^{*} / \mathrm{S}_{0} \mathrm{CI}$, our measurement reflects the exit of the ionization window, which, as already explained, takes place at shorter $\mathrm{N}-\mathrm{H}$ distances, around $1.3 \AA{ }^{48}$ Additionally, the values form Lippert et al. are affected by a considerably large error of $80 \mathrm{fs}$, attributed to the uncertainty of the fitting procedure and to the limited time resolution of the experiment.

The dynamics measured after direct excitation of the $\pi \sigma^{*}$ state can also be examined on the light of the wavepacket dynamical calculations carried out by Vallet et $a l .{ }^{37}$ The population probabilities on the $\pi \sigma^{*}$ surface calculated at CASSCF/aug-ccpVDZ level decay on the order of hundreds of fs, when the state is prepared at the ground level of the $\mathrm{NH}$ stretch tuning mode. This rate reflects the existence of a small well at the $\pi 3 \mathrm{~s}$ part of the potential that the wavepacket escapes by tunneling, slowing down the dynamics along the $\pi \sigma^{*}$ surface. The lifetimes measured in our experiments at all the excitation wavelengths, including the direct population of the $\pi \sigma^{*}$ at $252 \mathrm{~nm}$, do not seem to support this picture, corresponding to a barrierless process. In addition, the time dependent calculations carried out agree with the experimental data, by showing a small barrier below the ground level of the NH stretch vibration.

Another aspect of the problem that deserves further discussion is the IC of the upper $\pi \pi^{*}$ state when excited adiabatically off-resonance, or resonantly at $217 \mathrm{~nm}$. Barbatti et al. ${ }^{38}$ have investigated the photochemical deactivation pathways of pyrrole involving the upper $\pi \pi^{*}$ state by means of multireference configuration interaction calculations. According to this study, the out-of-plane deformation mode of the pyrrole ring creates a barrier-less pathway along the ${ }^{1} \mathrm{~B}_{2} \pi \pi^{*}$ surface that crosses the $\pi \sigma$ and the ground state $\mathrm{S}_{0}$, forming conical intersections. This relaxation channel connects consecutively the initially excited $\pi \pi^{*}$ state with the $\pi \sigma^{*}$ and the ground state. The dynamical behavior observed after exciting the $\pi \pi^{*}$ state at $217 \mathrm{~nm}$ can be explained according to these theoretical findings. The prepared $\pi \pi^{*}$ state converts to the lower $\pi \sigma^{*}$ with the $\tau_{22}=19 \pm 3$ lifetime, presumably through the predicted $\pi \pi^{*} / \pi \sigma^{*}$ CI promoted by the ring deformation mechanism. Once at the $\pi \sigma^{*}$ surface, the system evolves along the $\mathrm{NH}$ stretch coordinate giving rise to the already discussed $\tau_{33}$ lifetime.

It may be interesting to compare the photophysics of pyrrole with the role the $\pi \sigma^{*}$ state plays in other simple chromophores. The relaxation mechanisms involving this state have been previously tracked in aniline ${ }^{19}$ and indole, ${ }^{11,12}$ 
showing a quite distinct behavior. In indole and aniline the dynamics along the $\pi \sigma^{*}$ surface has been measured to occur in hundreds of femtoseconds, considerably slower than in pyrrole, which can be explained by the existence of a deeper well along the NH stretching coordinate. However, what mainly determines the available photochemical and photophysical relaxation pathways is the relative energy of the $\pi \sigma^{*}$ and $\pi \pi^{*}$ states. On the contrary to pyrrole where the $\pi \sigma^{*}$ is the lowest excitation, in the case of aniline and indole the $\pi \sigma^{*}$ state minimum is located above at least one of the $\pi \pi^{*}$ excitations. As a consequence the observed dynamics is completely different. While in pyrrole the relaxation is mediated by the $\pi \sigma^{*}$ state and the molecule survives electronically excited for tens of femtoseconds (triplet state participation is neglected), aniline and indole, even when excited above the $\pi \pi^{*} / \pi \sigma^{*}$ crossing point, show a long term picoseconds relaxation that involves the lowest $\pi \pi^{*}$ state. The later situation points to a more complicated relaxation mechanism, in which the activation of the proper vibrational mode to access the $\pi \sigma^{*}$ surface, together with the vibrational redistribution processes, likely to play a central role.

\section{CONCLUDING REMARKS}

The carried out experiments have revealed a rich femtosecond dynamics in the pyrrole isolated molecule, when excited at the near portion of its UV absorption spectrum. The transients recorded in the 264-217 nm excitation range show a $15 \pm 3$ fs decay that is attributed to dynamics on the dissociative surface of a $\pi \sigma^{*}$ character state, the lowest excited state of the molecule. This lifetime reflects the loss of ionization cross-section caused by the wavepacket movement along the repulsive $\mathrm{NH}$ coordinate. The rate of the process, 15 $\pm 3 \mathrm{fs}$, indicates that the system reaches the exit of the ionization window without crossing any barrier. Although the experiments do not show the system evolution after this point, the measured dynamics can be correlated with previous observations regarding the ultrafast formation of $\mathrm{H}$ atoms. ${ }^{31,33,36}$

The work also reveals the mechanisms that control the population of the $\pi \sigma^{*}$ surface. Regarding this aspect, a key idea introduced by the study is the possibility of coherent outof-resonance excitation of the molecule. The striking observation of the $\pi \sigma^{*}$ state dynamics at excitation wavelengths to the red of the lowest electronic transition can only be understood, in terms of the out-of-resonance excitation of the intense $\pi \pi^{*}$ transition, and subsequent internal conversion toward the lower $\pi \sigma^{*}$ state during the adiabatic evolution of the $\pi \pi^{*}$-laser interaction. As a result, the modelling of the system molecular response requires the use of coherent treatment, which describes the time dependence of the $\pi \pi^{*}$ and $\pi \sigma^{*}$ states in terms of the optical Bloch equations. In the model, the $\pi \sigma^{*}$ state is essentially populated by $\pi \sigma^{*} \leftarrow \pi \pi^{*}$ IC, being the rate of the process $\tau_{22}=19 \pm 3 \mathrm{fs}$. This state is also found to be accessible by direct excitation, presenting a small relative oscillator strength with respect to the $\pi \pi^{*}$ absorption: $\mathrm{f}_{\pi \sigma^{*}} / \mathrm{f}_{\pi \pi^{*}} \sim 0.0027$.

The carried out research substantiates the importance of the non-resonant adiabatic excitation when coherent femtosecond pulses are employed to trigger the electronic excita- tion of the molecule. In the case of pyrrole, although the $\pi \pi^{*}$ state absorption starts at $220 \mathrm{~nm}$, this effect provides a route to populate the dissociative $\pi \sigma^{*}$ at wavelengths as long as $264 \mathrm{~nm}$. Consequently, the study of isolated systems by coherent sources of increasing time resolution is required to consider a great portion of the electronic spectrum, to properly understand the relaxation mechanisms. This effect also represents a nice example on how the interaction with the excitation pulse not only triggers the process, but it may determine the temporal evolution of the system.

\section{ACKNOWLEDGMENTS}

This study was funded by Spanish MICINN (Grant No. CTQ2010-17749) and Consolider Program "Science and Applications of Ultrafast Ultraintense Lasers" (Grant No. CSD2007-00013), and by the Basque Government through the "Ayudas para apoyar las actividades de grupos de investigación del sistema universitario vasco" program. The experiments and theoretical calculations were carried out at the SGIker laser facility, and IZO-SGI of the UPV/EHU, respectively.

${ }^{1}$ R. A. Jones, E. C. Taylor, and A. Weissberger, Pyrrole: The Chemistry of Heterocyclic Compounds (Wiley, New York, 1990).

${ }^{2}$ A. L. Sobolewski and W. Domcke, Chem. Phys. Lett. 321, 479 (2000); A. Kumar, M. Kołaski, and K. S. Kim, J. Chem. Phys. 128, 034304 (2008).

${ }^{3}$ A. L. Sobolewski, W. Domcke, C. Dedonder-Lardeux, and C. Jouvet, Phys. Chem. Chem. Phys. 4, 1093 (2002).

${ }^{4}$ M. N. R. Ashfold, B. Cronin, A. L. Devine, R. N. Dixon, and M. G. D. Nix, Science 312, 1637 (2006).

${ }^{5}$ A. L. Sobolewski and W. Domcke, J. Phys. Chem. A 111, 11725 (2007).

${ }^{6}$ M. N. R. Ashfold, A. L. Devine, R. N. Dixon, G. A. King, M. G. D. Nix, and T. A. A. Oliver, Proc. Natl. Acad. Sci. U.S.A. 105, 12701 (2008).

${ }^{7}$ B. C. Dian, A. Longarte, and T. S. Zwier, J. Chem. Phys. 118, 2696 (2003).

${ }^{8}$ M. Lin, C. Tseng, Y. T. Lee, and C. Ni, J. Chem. Phys. 123, 124303 (2005).

${ }^{9}$ M. G. D. Nix, A. L. Devine, B. Cronin, and M. N. R. Ashfold, Phys. Chem. Chem. Phys. 8, 2610 (2006).

${ }^{10}$ A. Iqbal and V. G. Stavros, J. Phys. Chem. A 114, 68 (2010).

${ }^{11}$ R. Livingstone, O. Schalk, A. E. Boguslavskiy, G. Wu, L. T. Bergendahl, A. Stolow, M. J. Paterson, and D. Townsend, J. Chem. Phys. 135, 194307 (2011).

${ }^{12}$ R. Montero, A. Peralta Conde, V. Ovejas, F. Castaño, and A. Longarte, J. Phys. Chem. A 116, 2698 (2012).

${ }^{13}$ L. Serrano-Andrés and B. O. Roos, J. Am. Chem. Soc. 118, 185 (1996).

${ }^{14}$ M. G. D. Nix, A. L. Devine, B. Cronin, R. N. Dixon, and M. N. R. Ashfold, J. Chem. Phys. 125, 133318 (2006).

${ }^{15}$ G. M. Roberts, A. S. Chatterley, J. D. Young, and V. G. Stavros, J. Phys. Chem. Lett. 3, 348 (2012).

${ }^{16}$ T. Ebata, C. Minejima, and N. Mikami, J. Phys. Chem. A 106, 11070 (2002).

${ }^{17}$ Y. Honda, M. Hada, M. Ehara, and H. Nakatsuji, J. Chem. Phys. 117, 2045 (2002).

${ }^{18}$ G. A. King, T. A. A. Oliver, and M. N. R. Ashfold, J. Chem. Phys. 132, 214307 (2010)

${ }^{19}$ R. Montero, A. Peralta Conde, V. Ovejas, R. Martínez, F. Castaño, and A. Longarte, J. Chem. Phys. 135, 054308 (2011).

${ }^{20}$ A. L. Devine, B. Cronin, M. G. D. Nix, and M. N. R. Ashfold, J. Chem. Phys. 125, 184302 (2006).

${ }^{21}$ M. Barbatti, H. Lischka, S. Salzmann, and C. M. Marian, J. Chem. Phys. 130, 034305 (2009).

${ }^{22}$ D. J. Hadden, K. L. Wells, G. M. Roberts, L. T. Bergendahl, M. J. Paterson, and V. G. Stavros, Phys. Chem. Chem. Phys. 13, 10342 (2011).

${ }^{23}$ R. Crespo-Otero, M. Barbatti, H. Yu, N. L. Evans, and S. Ullrich, ChemPhysChem 12, 3365 (2011).

${ }^{24}$ H. Yu, N. L. Evans, V. G. Stavros, and S. Ullrich Phys. Chem. Chem. Phys. 14, 6266 (2012).

${ }^{25}$ M. H. Palmer and P. J. Wilson, Mol. Phys. 101, 2391 (2003). 
${ }^{26}$ O. Christiansen, J, Gauss, J. F. Stanton, and P. Jørgensen, J. Chem. Phys. 111, 525 (1999).

${ }^{27}$ J. Van, J. Meller, M. Hada, M. Ehara, and H. Nakatsuji, J. Chem. Phys. 113, 7853 (2000).

${ }^{28}$ B. O. Roos, P. Malmqvist, V. Molina, L. Serrano-Andrés, and M. Merchán, J. Chem. Phys. 116, 7526 (2002).

${ }^{29}$ P. A. Mullen and M. K. Orloff, J. Chem. Phys. 51, 2276 (1969).

${ }^{30}$ M. H. Palmer, I. C. Walker, and M. F. Guest, Chem. Phys. 238, 179 (1998).

${ }^{31}$ J. Wei, J. Riedel, A. Kuczmann, F. Renth, and F. Temps, Faraday Discuss. 127, 267 (2004)

${ }^{32}$ J. M. Beames, M. G. D. Nix, and A. J. Hudson, J. Chem. Phys. 131, 174305 (2009).

${ }^{33}$ B. Cronin, M. G. D. Nix, R. H. Qadiri, and M. N. R. Ashfold, Phys. Chem. Chem. Phys. 6, 5031 (2004)

${ }^{34}$ B. Cronin, A. L. Devine, M. G. D. Nix, and M. N. R. Ashfold, Phys. Chem. Chem. Phys. 8, 3440 (2006)

${ }^{35}$ L. Rubio-Lago, D. Zaouris, Y. Sakellariou, D. Sofikitis, T. N. Kitsopoulos, F. Wang, X. Yang, B. Cronin, A. L. Devine, G. A. King, M. G. D. Nix, M. N. R. Ashfold, and S. S. Xantheas, J. Chem. Phys. 127, 064306 (2007).

${ }^{36}$ H. Lippert, H.-H. Ritze, I. V. Hertel, and W. Radloff, ChemPhysChem 5, 1423 (2004)

${ }^{37}$ V. Vallet, Z. Lang, S. Mahapatra, A. L. Sobolewski, and W. Domcke, Faraday Discuss. 127, 283 (2004).
${ }^{38}$ M. Barbatti, M, Vazdar, A. J. A. Aquino, M. Eckert-Maksić, and H. Lischka, J. Chem. Phys. 125, 164323 (2006).

${ }^{39}$ See supplementary material at http://dx.doi.org/10.1063/1.4742344 for an extended explanation on the UV generation set-up and the coherent evolution of a two-level system.

${ }^{40}$ M. J. Frisch, G. W. Trucks, H. B. Schlegel et al., Gaussian 09, Revision A.02, Gaussian, Inc., Wallingford, CT, 2009.

${ }^{41}$ S. Mukamel, Principles of Nonlinear Optical Spectroscopy (Oxford University Press, Oxford, 1995).

${ }^{42}$ A. Peralta Conde, R. Montero, A. Longarte, and F. Castaño, Phys. Chem. Chem. Phys. 12, 15501 (2010).

${ }^{43} \mathrm{~B}$. W. Shore, The Theory of Coherent Atomic Excitation (Wiley, New York, 1990).

${ }^{44}$ N. V. Vitanov, B. W. Shore, L. Yatsenko, K. Böhmer, T. Halfmann, T. Rickes, and K. Bergmann, Opt. Commun. 199, 117 (2001).

${ }^{45}$ A. Peralta Conde, L. Brandt, and T. Halfmann, Phys. Rev. Lett. 97, 243004 (2006).

${ }^{46}$ R. Montero, A. Peralta Conde, A. Longarte, and F. Castaño, ChemPhysChem. 11, 3420 (2010).

${ }^{47}$ A. Peralta Conde, P. Tzallas, and D. Charalambidis, Eur. Phys. J. D 51, 289 (2009).

${ }^{48}$ M. Barbatti and S. Ullrich, Phys. Chem. Chem. Phys. 13,15492 (2011). 